\title{
A genetic variant of the NTCP gene is associated with HBV infection status in a Chinese population
}

\author{
Jingmin Yang ${ }^{1,2+}$, Yuan Yang ${ }^{3,4+}$, Mingying Xia ${ }^{1,2}$, Lianghui Wang ${ }^{1,2}$, Weiping Zhou ${ }^{3,5}$, Yajun Yang ${ }^{1,2}$,
} Yueming Jiang ${ }^{1,2}$, Hongyang Wang ${ }^{3,4,5}$, Ji Qian ${ }^{6,7,1,2^{*}}$, Li Jin $^{1,2^{*}}$ and Xiaofeng Wang ${ }^{1,2^{*}}$

\begin{abstract}
Background: To investigate whether genetic variants of the HBV receptor gene NTCP are associated with HBV infection in the Han Chinese population.

Methods: We sequenced the entire $23 \mathrm{~kb}$ NTCP gene from 111 HBeAg-positive HBsAg carriers (PSE group), 110 HBeAg-negative HBsAg carriers (PS group), and 110 control subjects. Then, we performed association analyses of suggestively significant SNPs with HBV infection in 1075 controls, 1936 PSs and 639 PSEs.

Results: In total, 109 rare variants (74 novel) and 38 single nucleotide polymorphisms (SNPs, one novel) were screened. Of the seven non-synonymous rare variants, six were singletons and one was a double hit. All three damaging rare singletons presented exclusively in the PSE group. Of the five SNPs validated in all 3650 subjects, the T allele of rs4646287 was significantly decreased $(p=0.002)$ in the PS group (10.1\%) and PSE group (8.1\%) compared to the controls (10.9\%) and was decreased to $7.4 \%$ in the PSE hepatocellular carcinoma (HCC) subgroup. Additionally, rs4646287-T was associated with a 0.68-fold ( $95 \% C l=0.51-0.89, p=0.006$ ) decreased risk of PSE compared with the controls. The NTCP mRNA level was lower in HCC tissues in " $\mathrm{CT}+T$ " carriers than in "CC" carriers.
\end{abstract}

Conclusions: We found a genetic variant (rs4646287) located in intron 1 of NTCP that may be associated with increased risk of HBV infection in Han Chinese.

Keyword: HBV-receptor, NTCP, Genetic variant, HBV infection status, Association, rs4646287

\section{Background}

Hepatitis B virus (HBV) infection is a major global public health issue. Serological evidence of HBV infection was observed in approximately one-third of the world's population, of which 350-400 million people were chronically infected [1]. Individuals infected with HBV are at high risk for the development of cirrhosis, liver failure, or hepatocellular carcinoma $(\mathrm{HCC})[2,3]$. HBV is a DNA virus that

\footnotetext{
* Correspondence: jiqian05@gmail.com; lijin.fudan@gmail.com; xiaofengwang71@163.com

${ }^{\dagger}$ Equal contributors

${ }^{6}$ Cancer Institute, Fudan University Shanghai Cancer Center, Shanghai 200032, China

${ }^{1}$ Epidemiology unit of MOE Key Laboratory of Contemporary Anthropology and State Key Laboratory of Genetic Engineering, School of Life Sciences and Institutes of Biomedical Sciences, Fudan University, 220 Handan Rd., Shanghai 200433, China

Full list of author information is available at the end of the article
}

primarily infected hepatocytes which mediated by HBV receptors located on the hepatocyte cell membrane. Although identifying the HBV receptors is not easy, several candidates have been reported [4-6].

Recently, Li et al. found that NTCP (sodium taurocholate cotransporting polypeptide), which was specifically expressed in hepatocytes, was a necessary cell surface receptor for HBV entry [7]. NTCP specifically interacts with the receptor-binding region of HBV pre-S1 and mediates woolly monkey HBV infection of Tupaia hepatocytes [7, 8]. Silencing NTCP inhibited HBV and HDV infection, whereas exogenous NTCP expression rendered nonsusceptible hepatocarcinoma cells susceptible to viral infection [7]. Additionally, Dieter Glebeb et al. found that tent-making bat HBV (TBHBV) could infect human hepatocytes using the human NTCP (hNTCP) receptor [9].

\section{Biomed Central}


Host genetics play an important role in the clinical outcome of $\mathrm{HBV}$ infection. Due to the popularity of genome-wide association studies (GWAS) in recent years, several susceptibility loci associated with HBVrelated diseases have been identified. For example, genetic variants in the HLA-DP locus were found to be strongly associated with a risk of persistent HBV infection [10]. Several genetic susceptible loci for HBV-related HCC were also identified [11-13]. However, the relationship between genetic variants of full-length NTCP and the HBV infection status has rarely been investigated $[14,15]$. In this study, we investigated whether genetic variants in the NTCP gene predisposed subjects to $\mathrm{HBsAg}+\mathrm{HBeAg}$-positivity (representing higher HBV activity) and HBsAg-positivity (representing lower HBV activity) based on the finding that a higher serum HBV DNA concentration was observed in HBeAg-positive compared to $\mathrm{HBeAg-negative} \mathrm{indi-}$ viduals $[1,16,17]$.

\section{Methods}

\section{Subjects}

A population-based study wad designed with three groups for comparison, including 639 subjects positive for HBsAg and HBeAg (the PSE group), 1936 subjects positive for $\mathrm{HBsAg}$ (the PS group) and 1075 subjects negative for $\mathrm{HBV}$ antibodies and antigens (the control group). All of the study subjects were East China residents. A total of 2647 subjects were randomly selected from the hepatitis sub-cohort (including 43,000 subjects) of the Taizhou longitudinal (TZL) study. The profile of the TZL study was described elsewhere [18]. An additional 299 subjects with hepatocellular carcinoma for the PSE group and 704 subjects with hepatocellular carcinoma for the PS group (HCC, long-term outcome of persistent infection of $\mathrm{HBV}$ ) were recruited from the Shanghai Eastern Hepatobiliary Surgery Hospital (EHBH). The ages of the subjects in the PSE group, PS group, and control group were $45.34 \pm 11.55 \mathrm{yrs}, 52.36 \pm 11.35 \mathrm{yrs}$, and $53.53 \pm 12.93 \mathrm{yrs}$, respectively, and the three groups included 67.3, 65.6, and $49.5 \%$ males, respectively. For the functional analysis, 33 paired tumor/non-neoplastic adjacent (normal) liver tissues from HBV-related HCC patients were obtained from the EHBH. Written informed consent was acquired from all of the participants, and the study protocol was approved by the Ethics Committee of the School of Life Sciences of Fudan University.

\section{Identification of SNPs/indels in NTCP by Sanger sequencing DNA preparation}

Genomic DNA was isolated from human peripheral blood lymphocytes using the Mini DNA Extraction kit for Peripheral Blood (Lifefeng, Shanghai, China) and quantified with the NanoDrop ND-1000 spectrophotometer (NanoDrop, Wilmington, DE, USA).

\section{Primer design}

A full-length of 23,872 bp sequence of the human NTCP gene was obtained from the GENE database of NCBI (National Center for Biotechnology Information, RefSeq: NC_000014.8) for primer design. Overall, 42 pairs of PCR primers were designed to amplify this gene, including the full-length gene and the core promoter, located from $70,240,928$ to $70,264,606$ on chromosome 14 . The lengths of the PCR amplicons ranged from 725 bp to $1821 \mathrm{bp}$. The forward primers and/or reverse primers for each amplicon were used as Sanger sequencing primers. Furthermore, several nested primers were also used as the sequencing primers for the two long amplicons [Additional file 1].

\section{PCR-based Sanger sequencing of NTCP}

The PCR products of each primer pair were sequenced using an ABI 3730xl DNA Analyzer (Applied Biosystems, Foster City, CA, USA). Overall, 111, 110 and 110 subjects were chosen randomly from the PSE, PS, and control groups, respectively, for variant sequencing and variant frequency comparisons among groups.

\section{SNP and indel identification}

SNPs were identified utilizing the software CodonCode Aligner (CodonCode Corporation, Centerville, MA, USA), and indels were deduced manually after aligning to the reference sequence.

\section{SNP genotyping assay}

The variants for genotyping were chosen according to the following criteria: (1) SNPs distributed significantly $(p<0.05)$ or marginally significantly $(p<0.06)$ between any two groups (control, PS, PSE, and PS + PSE groups); (2) non-synonymous SNPs; (3) selected SNPs in the main LD block; and (4) novel variants that occurred only in the PSE + PS or controls at least three times.

The TaqMan SNP genotyping assay was performed to genotype the chosen variants in 528 PSE subjects, 1826 PS subjects and 965 Controls. All samples were genotyped using TaqMan on an ABI GeneAmp ${ }^{\circ}$ PCR system 9700 (Applied Biosystems, Foster City, CA, USA) and an ABI Prism 7900HT sequence detection system (Applied Biosystems, Foster City, CA, USA).

\section{Analysis of gene expression using real-time PCR}

Total RNA was extracted from paired tumour and normal liver tissues from HBV-related $\mathrm{HCC}$ patients with the TRIzol reagent (Invitrogen, Carlsbad, CA, USA) [19] according to the manufacturer's protocol. The concentration of the isolated RNA and the A260/A280 
ratio were measured with the NanoDrop ND-1000 spectrophotometer (NanoDrop Technologies, Wilmington, DE, USA). Then, $1 \mu \mathrm{g}$ of the total RNA was used to synthesize first-strand cDNA with the PrimeScript ${ }^{\mathrm{Tm}}$ RT reagent Kit (TaKaRa Bio, JPN). These products were diluted 1:40, and $2 \mu \mathrm{l}$ was used as the template in a $10 \mu \mathrm{l}$ real-time PCR reaction performed on an $\mathrm{ABI}$ Prism 7900 HT Sequence Detection System (Applied Biosystems, Foster City, CA, USA) using the SYBR ${ }^{\circledast}$ Premix Ex Taq ${ }^{\mathrm{TM}}$ II (TaKaRa Bio, JPN). The housekeeping gene $A C T B$ was adopted as the reference gene for the real-time PCR. Primers spanning the introns were designed using the Primer Express ${ }^{\mathrm{TM}}$ software (Applied Biosystems, Foster, CA, USA). The primer sequences were as follows:

ACTB: Forward (5'-GCTCCGGCATGTGCAA-3');

Reverse (5'TCGCCCACATAGGAATCCTT-3')

NTCP: Forward (5'-GGACATGAACCTCAGCATTG TG-3');

Reverse (5' -ATCATAGATCCCCCTGGAGTAGAT-3').

\section{Statistical analysis}

Deviation from the Hardy-Weinberg equilibrium (HWE) expectation for any genetic variant was tested using a Chisquare statistic. Continuous variables were expressed as the means ( \pm standard deviation), and comparisons of continuous variables were conducted using the Student's $t$-test. Unconditional logistic regression analyses were used to estimate the odds ratios (ORs) and their $95 \%$ confidence intervals (CIs) for PS (HBsAg-positivity) or PSE (HBsAg- and HBeAgpositivity) under the assumption of different genetic models. Multivariate analyses were performed to adjust for age and gender. SPSS software version 16.0 for Windows was used in the statistical evaluation of the above-mentioned data.

The NTCP mRNA expression levels were calculated using the $2^{-\Delta \mathrm{ct}}$ method. The NTCP mRNA expression levels were calculated relative to the ACTB housekeeping gene. The fold changes in the NTCP mRNA levels of tumor tissues relative to their paired normal tissues were calculated using the $2^{-\Delta \Delta c t}$ method.

To construct relevant haplotypes, the genotype data were used to estimate the inter-marker LD, measure the pair-wise D' and $\mathrm{r}^{2}$ and define the LD blocks. Haplotype inferences and haplotype association tests were estimated using Haploview [20].

Because the sample size was relatively small, a suggestive significance threshold of $\mathrm{P}<0.05$ was used to select SNPs for variant discovery. The Bonferroni correction was used to weed out false positive findings due to multiple testing. A significance threshold of $\mathrm{P}<0.01$ was used after multiplication by the total number of genotyped SNPs $(0.05 / 5)$.

\section{Results}

\section{Variants discovered by sequencing}

We sequenced $\sim 23 \mathrm{~kb}$ of the NTCP gene, including all five exons, four introns, as well as the core promoter region [21], and a $1.2 \mathrm{~kb}$ region downstream of the NTCP gene with high success rates. The exception was six regions $(\sim 3 \mathrm{~kb})$ located in the first and second introns around the highly repetitive elements (i.e., Alu repeats and homo-polymers). Overall, 147 variants, including 109 rare variants (74 novel) and 38 SNPs (one novel), were detected [see Additional file 2]. Approximately $91.8 \%$ (135) of the variants were located in nonexon regions, with $38.8 \%$ (30) in the first intron, $33.3 \%$ (22) in the second intron and $11.6 \%$ (17) in downstream (16) and upstream (1) regions. Moreover, the MAF (minor allele frequency) of $74.1 \%$ (109) and $15.0 \%$ (22) of the variants lied below $1 \%$ and ranged from 10 to $20 \%$, respectively [see Additional file 3].

Among the 10 variants in the coding sequence, eight were non-synonymous, and two were synonymous variants; six of the non-synonymous and one of the synonymous variants were singletons. The effects of non-synonymous coding variants on protein functions were predicted by SIFT [22] and PolyPhen [23] (http:// www.snp-nexus.org/) (Table 1). The variants 333A > T and $88 \mathrm{I}>\mathrm{T}$ (both of which were predicted to be tolerated/benign by SIFT/Polyphen) were not found in the PSE group. Correspondingly, three variants $(256 \mathrm{M}>\mathrm{T}$, $222 \mathrm{~L}>\mathrm{S}$, and $200 \mathrm{~V}>\mathrm{M}$ ) predicted to be damaging/ possibly damaging presented exclusively in the PSE group. Furthermore, a rare SNP 267S > F variant (rs2296651) that was predicted to be probably damaging by Polyphen and to cause a near complete loss of function for bile acid uptake of NTCP [24] occurred more frequently in the PSE group. Based on these observations, more damaging mutations were found in PSE group than the control or PS groups.

\section{SNP rs4646287 and HBV infection}

Based on the validation criteria described in the Materials and Methods, six SNPs from the identified variants were chosen for genotyping in all subjects. Among the six SNPs, SNP rs2296651 was a non-synonymous damaging variant. SNP rs4646287 exhibited difference between the PSE and control groups with marginal statistical significance. Nineteen common SNPs were used to construct a large haplotype block $\left(\mathrm{r}^{2}\right.$ ranged from 0.74 to 1 ) spanning approximately $21 \mathrm{~kb}$; three of these SNPs (rs4646285, rs28437822 and rs11624523) were chosen, of which rs4646285 was located in exon 1 . The rare variant +20988G > A occurred three times only in the PSE + PS group.

Because the homozygote mutant TT genotype frequency of rs4646287 was only $0.7,1.3$, and $1.2 \%$ in the control, 
Table 1 Non- synonymous variants identified in the sequencing results

\begin{tabular}{|c|c|c|c|c|c|c|c|c|c|c|}
\hline \multirow[t]{2}{*}{ Variants } & \multirow[t]{2}{*}{ AA change } & \multirow[t]{2}{*}{ Novel } & \multirow[t]{2}{*}{ Exon } & \multicolumn{2}{|c|}{ Sift Prediction } & \multicolumn{2}{|c|}{ Polyphen Prediction } & \multicolumn{3}{|l|}{ MAF (\%) } \\
\hline & & & & Score & Prediction & Score & Prediction & Control & PS & PSE \\
\hline$+21059 \mathrm{C} / \mathrm{T}$ & $333 \mathrm{~A}>\mathrm{T}$ & Yes & 5 & 0.28 & Tolerated & 0 & Benign & 0 & 0.5 & 0 \\
\hline$+21029 \mathrm{C} / \mathrm{G}$ & $323 A>P$ & Yes & 5 & 0.08 & Tolerated & 0.476 & Possibly damaging & 0.5 & 0 & 0 \\
\hline rs2296651 & $267 S>F$ & No & 4 & 0.31 & Tolerated & 0.88 & Probably damaging & 0.9 & 0.9 & 2.3 \\
\hline +18885 A/G & $256 \mathrm{M}>\mathrm{T}$ & Yes & 4 & 0 & Damaging & 0.839 & Possibly damaging & 0 & 0 & 0.5 \\
\hline +18131 A/G & $222 \mathrm{~L}>\mathrm{S}$ & Yes & 3 & 0 & Damaging & 0.637 & Possibly damaging & 0 & 0 & 0.5 \\
\hline rs202213974 & $200 V>M$ & No & 3 & 0 & Damaging & 0.819 & Possibly damaging & 0 & 0 & 0.5 \\
\hline$+11121 \mathrm{G} / \mathrm{C}$ & $131 \mathrm{~L}>\mathrm{V}$ & Yes & 2 & 0.23 & Tolerated & 0.484 & Possibly damaging & 0.5 & 0 & 0 \\
\hline rs148467625 & $881>T$ & No & 1 & 0.57 & Tolerated & 0.072 & Benign & 0.5 & 0.5 & 0 \\
\hline
\end{tabular}

PS, and PSE groups, respectively, we combined the rare homozygotes and heterozygotes for further analysis in a dominant model. The $\mathrm{T}$ allele was associated with a decreased risk of PSE with a MAF of $8.1 \%$ compared with the control group that had a MAF of $10.9 \%(O R=0.67$, 95 \% CI: 0.51-0.87, $p=0.003$, Table 2). The association remained after adjusting for age and gender $(O R=0.68$, $95 \%$ CI (0.51-0.79), $p=0.006)$. Additionally, the $\mathrm{T}$ allele frequencies decreased significantly from the Control (10.9\%), to the HCC (-) PS (10.3\%) subgroup, HCC (+) PS subgroup (9.7\%), HCC (-) PSE (8.7\%) subgroup and HCC (+) PSE subgroup $(7.4 \%)\left(p_{\text {linear }}=0.002\right)$ (Fig. 1).

NTCP expression differences among rs4646287 genotypes To evaluate the functional effects of rs4646287 on the transcriptional activity of NTCP, 33 paired tumor/normal liver tissues from HCC patients (16 $\mathrm{CC}, 15 \mathrm{CT}$, and $2 \mathrm{TT}$ genotypes) were recruited to measure the NTCP mRNA levels using the real-time fluorescence quantitative PCR method. The clinical information for the samples was provided in Additional file 4. NTCP mRNA levels were lower in subjects with $\mathrm{CT}+\mathrm{TT}$ genotypes than those with the CC genotype, although the difference was nearly 4.8 fold in tumor tissues but only $10 \%$ in normal tissues (Fig. 2, Additional file 5).

\section{Other SNPs and HBV infection}

We validated three SNPs (rs4646285, rs11624523, and rs28437822) among the 19 SNPs used to construct the large LD block. However, non of these SNPs exhibited a significant difference among the PSE, PS, and control groups. The non-synonymous SNP rs2296651 resulted in an amino acid change from serine to phenylalanine at position $267(267 \mathrm{~S}>\mathrm{F})$ of the NTCP protein. The frequencies of the A allele were 2.67, 2.57, and $4.15 \%$ in the control, PS, and PSE groups, respectively. However, we did not find any significant differences in the A allele frequency among the three groups after adjusting for multiple testing.
The rare variant $+20988 \mathrm{G}>\mathrm{A}$ occurred only in the PSE + PS group

The variant $+20988 \mathrm{G}>\mathrm{A}$ located in intron 4, $18 \mathrm{bp}$ upstream of exon 5 arose only in the PSE + PS subjects. This variant occurred twice in the PS group and once in the PSE group among the sequenced subjects and five times in the PS group and twice in the PSE group in the genotyped subjects. In contrast, it was not observed in the controls.

\section{Discussion}

In the present work, we described a genetic profile of the NTCP gene in a normal population, a HBe-negative population, and a HBe-positive population of Han Chinese. The rare and common genetic variants identified in the NTCP gene call for additional functional and trait association analyses. A functional genetic variant of NTCP (rs4646287) that was found to be associated with HBV infection status. The rare T allele of the SNP rs4646287 was not only linearly decreased in frequency in the control, PS, PSE and HCC subgroups but also predisposed to a decreased risk for PSE, which was indicative of reduced HBV activity. This result suggests a protective effect for the rs4646287-T allele for the host in response to $\mathrm{HBV}$ infection.

For the persistently infected patients in our study, the frequency of rs4646287 TT (or AA in the forward strand) was observed to be reduced (although not to a significant level) in the HCC group compared to the non-HCC group $[10(1.0 \%)$ vs. 22 (1.4 \%), $p=0.47$ under a recessive model]. Consistent with this finding, the frequency of the TT genotype was $0.7 \%$ in the HCC group and $1.4 \%$ in the non-HCC group in a GWAS study (data not published). However, the frequency of the rs4646287 TT genotype was slightly higher in the $\mathrm{HCC}$ group than in the non-HCC group in a study that included $186 \mathrm{HCC}$ and 395 non-HCC subjects [6 (3.2\%) vs. $1(0.3 \%)$ subjects, $p=0.018$ in a recessive model][14]. This discrepancy could be explained by the relatively small sample size or the different construction of the 
Table 2 SNPs associated with PSE and their odds ratios relative to the control

\begin{tabular}{|c|c|c|c|c|c|c|c|}
\hline \multirow[t]{2}{*}{ SNP } & \multirow{2}{*}{$\begin{array}{l}\text { Control, } \\
N=1075(100 \%)\end{array}$} & \multirow{2}{*}{$\begin{array}{l}P S_{1} \\
N=1936(100 \%)\end{array}$} & \multirow{2}{*}{$\begin{array}{l}\text { PSE, } \\
639(100 \%)\end{array}$} & \multirow[t]{2}{*}{$P_{\text {linear }}$} & \multicolumn{3}{|c|}{ PSE vs Control } \\
\hline & & & & & $P$ & OR & $95 \%$ Cl \\
\hline \multicolumn{8}{|l|}{ rs4646287 } \\
\hline CC & $843(79.0)$ & $1548(81.2)$ & $541(84.9)$ & & 0.005 & 1 & \\
\hline$C T$ & $215(20.1)$ & $334(17.5)$ & $89(14.0)$ & & 0.0014 & 0.65 & $0.49-0.85$ \\
\hline$\pi$ & $9(0.8)$ & $25(1.3)$ & $7(1.1)$ & & 0.705 & 1.21 & $0.45-3.27$ \\
\hline$C T+\pi$ & $224(21.0)$ & 359 (18.8) & $96(15.1)$ & 0.004 & 0.0026 & 0.67 & $0.51-0.87$ \\
\hline C & 1901 (89.1) & $3430(89.9)$ & 1171 (91.9) & & & 1 & \\
\hline T & $233(10.9)$ & $384(10.1)$ & $103(8.1)$ & 0.010 & 0.0075 & 0.72 & $0.56-0.92$ \\
\hline \multicolumn{8}{|l|}{ rs2296651 } \\
\hline GG & $1010(94.7)$ & $1834(95.2)$ & $585(91.7)$ & & & 1 & \\
\hline GA & $57(5.3)$ & $92(4.8)$ & $53(8.3)$ & 0.033 & 0.017 & 1.61 & $1.09-2.37$ \\
\hline G & 2077 (97.3) & 3760 (97.6) & $1223(95.8)$ & & & 1 & \\
\hline A & $57(2.7)$ & $92(2.4)$ & $53(4.2)$ & 0.036 & 0.021 & 1.58 & $1.05-2.35$ \\
\hline \multicolumn{8}{|l|}{ rs28437822 } \\
\hline AA & 769 (72.1) & $1383(72.2)$ & 470 (73.7) & & 0.062 & 1 & \\
\hline$A G$ & $266(25.0)$ & 491 (25.6) & $161(25.2)$ & & 0.933 & 0.99 & $0.79-1.24$ \\
\hline GG & $31(2.9)$ & $41(2.1)$ & $7(1.1)$ & & 0.018 & 0.37 & $0.16-0.85$ \\
\hline$A G+G G$ & $297(27.9)$ & $532(27.7)$ & $168(26.3)$ & 0.542 & 0.493 & 0.93 & $0.74-1.16$ \\
\hline A & 1804 (84.6) & 3257 (85.0) & 1101 (86.3) & & & 1 & \\
\hline G & $328(15.4)$ & $573(15.0)$ & $175(13.7)$ & 0.208 & 0.195 & 0.87 & $0.71-1.07$ \\
\hline \multicolumn{8}{|l|}{ rs11624523 } \\
\hline $\mathrm{AA}$ & 766 (71.5) & $1380(72.0)$ & 467 (73.7) & & 0.153 & 1 & \\
\hline$A G$ & $272(25.4)$ & $494(25.8)$ & $157(24.8)$ & & 0.638 & 0.95 & $0.75-1.19$ \\
\hline GG & $33(3.1)$ & $43(2.2)$ & $10(1.6)$ & & 0.056 & 0.50 & $0.24-1.02$ \\
\hline$A G+G G$ & $305(28.5)$ & $537(28.0)$ & $167(26.3)$ & 0.372 & 0.341 & 0.90 & $0.72-1.12$ \\
\hline A & 1804 (84.2) & 3254 (84.9) & 1091 (86.0) & & & 1 & \\
\hline G & 338 (15.8) & $580(15.1)$ & $177(14.0)$ & 0.110 & 0.166 & 0.87 & $0.71-1.06$ \\
\hline \multicolumn{8}{|l|}{ rs4646285 } \\
\hline CC & 768 (72.2) & 1391 (72.5) & $469(73.7)$ & & 0.051 & 1 & \\
\hline$C T$ & $264(24.8)$ & $486(25.3)$ & $160(25.2)$ & & 0.948 & 0.99 & $0.79-1.25$ \\
\hline$\pi$ & $32(3.0)$ & $42(2.2)$ & $7(1.1)$ & & 0.015 & 0.36 & $0.16-0.82$ \\
\hline$C T+\pi$ & $296(27.8)$ & $528(27.5)$ & $174(27.3)$ & 0.736 & 0.484 & 0.92 & $0.74-1.15$ \\
\hline$C$ & 1800 (84.6) & $3268(85.1)$ & $1098(86.3)$ & & & 1 & \\
\hline $\mathrm{T}$ & $328(15.4)$ & $570(14.9)$ & $174(13.7)$ & 0.182 & 0.178 & 0.87 & $0.71-1.06$ \\
\hline
\end{tabular}

aforementioned study. Indeed, the TT genotype was observed to be more common in the HCC group than in the non-HCC group [4 (1.3\%) vs. $3(0.9 \%)$ ] in the HBeAg-positive subjects (PSE group) in our study, although the difference did not reach statistical significance.

The SNP rs4646287, which is located in the first intron approximately $1 \mathrm{~kb}$ downstream of the first exon, may be an important variant that influences NTCP expression. Genetic variants in the first intron may be important for the predisposition of complex traits. These variants may be located in an intron-based transcription enhancer or silencer, with different alleles corresponding to different gene expression levels [25-27]. Using the transcriptional factor binding site prediction software TFSEARCH [28], we found that $\mathrm{C}>\mathrm{T}$ introduced a new DNA binding site for p300 (5' -AGGAGCG-3' to 5' -AG GAGTG-3'), which might influence the expression of $N T C P$ and suggested a functional significance for this variant. However, "CC" carriers exhibited increased NTCP mRNA levels compared to "CT + TT" carriers in normal tissues, and the lack of significance observed for this 
MAFs of rs 4646287

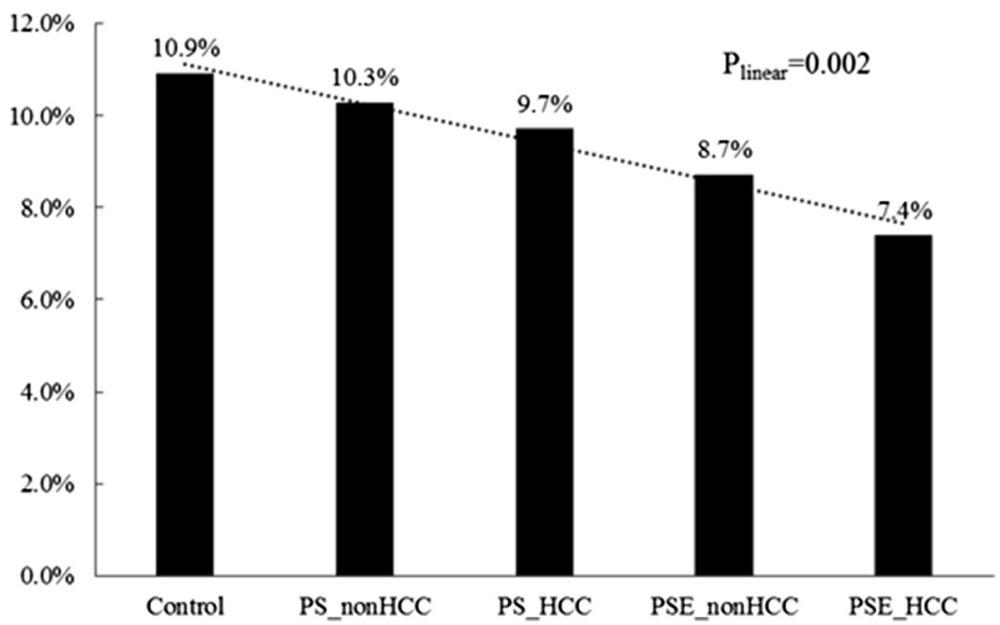

Fig. 1 MAFs of rs4646287 in different groups or subgroups

difference might be limited by the small sample size of our functional analysis $(n=33)$. Interestingly, the decreased NTCP mRNA levels of "CT + TT" compared to "CC" carriers reached as low as 0.21 -fold in the tumor tissues, which may be due to the complicated enlargement effects of the pathological status.

The potential functional variant rs4646287 that influenced the HBV infection status might have evolutionary implications. The frequencies of the rs4646287 $\mathrm{T}$ allele differed among different ethnic groups; the frequency was $0 \%$ in Africans and Europeans, but the allele was

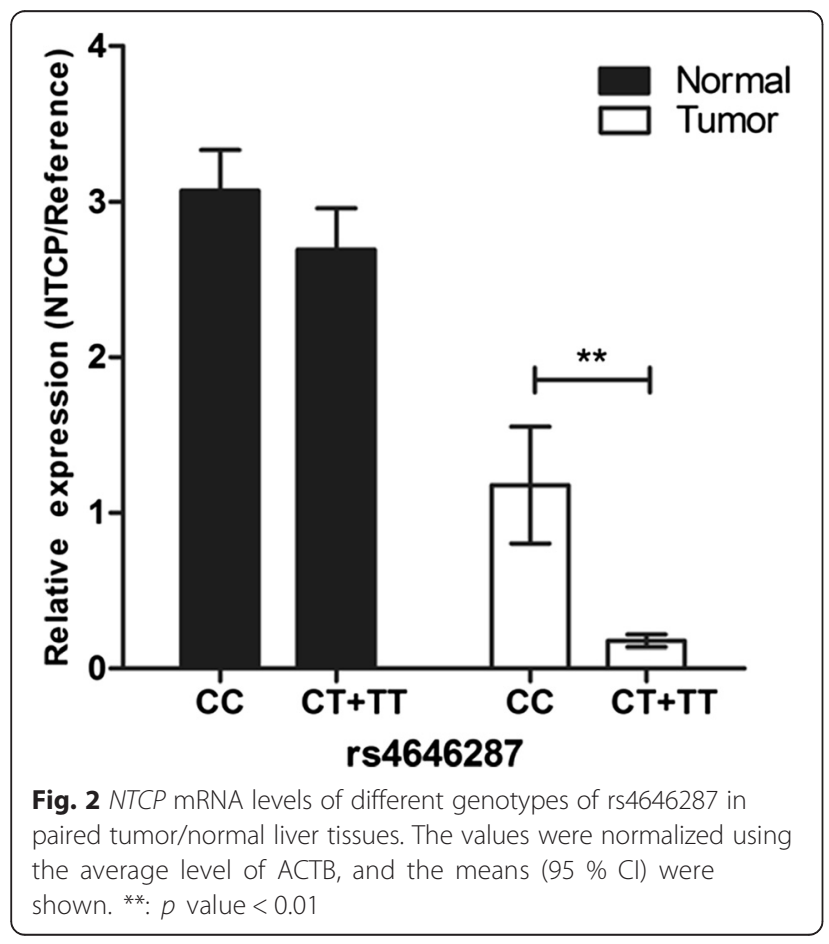

common in East Asians (11.31 \% in Chinese and $12.35 \%$ in Japanese populations [frequency information was obtained from the HapMap population data] and $10.93 \%$ in our control Han subjects). However, the frequencies of the $\mathrm{T}$ allele in the HBV-infected subjects decreased to $10.10 \%$ (PS group) and further decreased to $8.08 \%$ in subjects with a more severe status (PSE group). Because Asians are more likely to be infected with HBV, the frequency of the functional rs4646287-T was decreased in the control, PS, and PSE subjects. Because rs4646287-T decreases the expression and hence function of NTCP, these results suggest that $\mathrm{rs} 4646287-\mathrm{T}$ is a protective factor in response to the selective pressure of HBV infection in Asians that arose during the long process of human evolution.

Notably, although the increased presence of the rs2296651 (Ser267Phe) mutant allele in the PSE group compared to the PS or control group did not survive multiple testing correction, this SNP was worth particular consideration. Phe267 is differentially distributed in different ethnic groups, with $0 \%$ detected in Africans and Caucasians and a frequency of approximately 3-4\% in East Asians. Interestingly, the frequency of Phe267 decreases from southern to northern Chinese population, with $8.3 \%$ in Guangdong and $8.1 \%$ in Guangxi province (Southern China), $7.5 \%$ in Chongqing, $5.4 \%$ in Hunan and Hubei provinces, and $2.4 \%$ in Henan province in a cohort of hepatitis B patients undergoing therapy with interferon a2a (data not shown). Functional analysis revealed that the mutant allele had a deleterious effect on the transport of bile acids [24]. Moreover, this mutation also reduced the chance of HBV entering hepatocytes [29]. This observation indicated that the mutant allele might be lower in HBV carriers, which was confirmed by the study of Yiming Wang et. al. who 
found that the Ser267Phe variant was significantly associated with resistance to CHB [15]. In the present study, we found that the frequency of the mutant $\mathrm{A}$ allele was decreased in the PS group and increased in the PSE group relative to the controls. This finding was interesting, although we did not find an association between the Ser267Phe variant and the lower risk of $\mathrm{CHB}$, possibly due to the lower frequency of Ser267Phe in our population (Eastern China, $\mathrm{MAF}_{\text {control }}=2.7 \%$ ) compared to Yiming Wang's population (Southern China, $\mathrm{MAF}_{\text {control }}=10.9 \%$ ) [15]. The frequency of HBV genotype $C$ was reported to be higher in the PSE group than in the PS group in Han Chinese [30, 31]. Therefore, questions remain concerning whether the more invasive HBV-C prefers human rs2296651-A, resulting in a higher $\mathrm{A}$ in the PSE group. Our data showed that individuals with the rs2296651 GA genotype had a higher risk of infection with HBV-C than individuals with the GG genotype $(O R=1.42 p=0.75)$, although this difference did not reach statistical significance. Thus, this mutant allele might affect the interaction between HBV and its receptor NTCP.

Rare variants (especially damaging variants predicted by SIFT/PolyPhen) are more frequently presented in the PSE group. Three of the eight missense variants predicted to be damaging, including Met256Thr $(+18885$ A/G, novel) in the fourth exon and Leu222Ser $(+18131$ A/G, novel) and Val200Met (rs202213974) in the third exon, were observed only in the PSE group. Neighbouring variants may induce several missense mutations that significantly reduce the uptake of bile acid and/or estrone sulfate by NTCP [24]. These mutations include Ile223Thr (rs61745930) in the third exon and Ser267Phe (rs2296651), Ile279Thr (rs72547507) and Lys314Glu (rs72547506) in the fourth exon. The above observations may imply that the third or fourth exon of NTCP plays an important role in NTCP substrate transport and that missense mutations in these regions can easily have deleterious impacts on the transport function of NTCP. Similarly, the Leu222Ser, Val200Met, and Met256Thr variants discovered in the present study may lead to reduced bile acid transport activity. Coincidently, rare variants identified in other exons $(131 \mathrm{~L}>\mathrm{V}$ in exon 2, $88 \mathrm{I}>\mathrm{T}$ in exon 1 , and $323 \mathrm{~A}>\mathrm{P}$ and $333 \mathrm{~A}>\mathrm{T}$ in exon 5 ) predicted to be benign/tolerated were not accumulated in the third or fourth exons. Larger studies are needed to evaluate the pathological effects of these damaging variants on HBV predisposition. The novel rare variant $+20988 \mathrm{G}>\mathrm{A}$, which was adjacent to the boundary of the splicing site between intron 4 and exon 5, occurred 10 times in the PSE + PS group and was absent in the controls. This mutant might affect the splicing efficiency of NTCP.

To summarize, we recruited 1075 population-based healthy control subjects who did not carry antibodies and antigens against HBV, although this was not easy, due to the popularity of the hepatitis $B$ vaccine in China over the past 20 years. Using a special study design that included control, PS, PSE, and HCC groups, we failed to observe a dose effect of genetic variants on different traits but observed a trend towards the effects of genetic variants on different levels of the HBV infection status. However, the statistical power of the present study may be insufficient for variants with low frequencies.

\section{Conclusions}

In this study, we found that the genetic variant rs4646287 located in intron 1 of NTCP was associated with the HBV infection status in a Han Chinese population. Additionally, the coding variants discovered in the sequenced subjects (especially the race-specific SNP rs2296651) may help elucidate the relationship between NTCP substrate transport and HBV binding and provide a candidate for functional analysis. More studies on functional analysis of these mutations are needed to determine whether our finding is useful for HBV drug target identification.

\section{Availability of supporting data}

All primers used for Sanger sequencing [see Additional file 1] and variants reported in the manuscript [see Additional file 2] are provided in the Additional files.

\section{Additional files}

\begin{abstract}
Additional file 1: PCR and Sequencing primers in NTCP sequencing The list of all primers used in PCR and sequencing in this manuscript, including the sequences, amplicons' length and so on. (DOC $69 \mathrm{~kb}$ )
\end{abstract}

Additional file 2: Information of 147 Variations observed in all 331 sequenced subjects. The list of all variations observed in all 331 sequenced subjects in this manuscript, including the locations, positions, observed minor allele frequencies and so on. (DOC $227 \mathrm{~kb}$ )

Additional file 3: Distribution of Variants in NTCP in different groups. The MAFs of variants of each group (except for rs36115704 which has a much higher MAF of $49.6 \%$ ) show in different colors and symbols along their gene position: symbol "O" in black color, "*" in cyan color and "•" in red color represent MAFs of control group, PS group and PSE group, respectively. The $x$ axis indicates the physical position of the variants relative to the transcriptional initiation site $(+1)$, and the $y$ axis shows the values of within-group MAFs. The five colored boxes on the button of the plot represent the five exons of NTCP gene. The UTR regions show differently in blue with the coding regions show in orange. (TIFF $323 \mathrm{~kb}$ )

Additional file 4: Clinical Characteristics and Outcome of 33 HBV-relative HCC patients according to the genotypes of rs4646287. Clinical Characteristics and Outcome of 33 HBV-relative HCC patients according to the genotypes of rs4646287 were decripted in this table. No significant differences of these clinical characteristics were observed between the two genotype groups of rs4646287. (DOC $40 \mathrm{~kb}$ )

Additional file 5: NTCP mRNA expression levels in different genotypes of rs4646287. NTCP mRNA levels were lower in subjects with CT + TT genotypes than those with the CC genotype, although the difference was nearly 4.8 fold in tumor tissues but only $10 \%$ in normal tissues. (DOC $50 \mathrm{~kb}$ ) 


\section{Abbreviations}

PS: HBeAg-negativity HBsAg carriers; PSE: HBeAg-positivity HBsAg carriers; HCC: hepatocellular carcinoma; HBV: hepatitis B virus; SNP: single nucleotide polymorphisms; NTCP: sodium taurocholate cotransporting polypeptide; TBHBV: tent-making bat HBV; TZL: Taizhou longitudinal; MAF: minor allele frequency.

\section{Competing interests}

The authors declare that they have no competing interests.

\section{Authors' contributions}

$\sqcup$ and XFW designed the study. JMY and XFW wrote and edited the manuscript. YY, WPZ and HYW reviewed the specimen histology and contributed the HCC specimens. JQ, YJY and YMJ contributed the cohort samples. LHW and JMY isolated and purified the DNA and RNA from the clinical specimens and performed and analysed the GPCR data. JMY and MYX designed the primers. MYX, LHW and JMY directed the sequencing. All authors read and approved the final manuscript.

\section{Acknowledgements}

We thank all of the members who collected the samples and all of the subjects who participated in this study. This work was supported by grants from the Ministry of Science and Technology (2011BAI09B02), Natural Science Foundation of China (311712160, 81071681, and 81201940), Ministry of Health (201002007), National Basic Research Program (2012CB944600), State Key Infectious Disease Project of China (2012ZX10002010), National High-Tech Research and Development Program (2012AA021802), and International Cooperation Project of the Ministry of Science and Technology (2014DFA32830)

\section{Author details}

${ }^{1}$ Epidemiology unit of MOE Key Laboratory of Contemporary Anthropology and State Key Laboratory of Genetic Engineering, School of Life Sciences and Institutes of Biomedical Sciences, Fudan University, 220 Handan Rd., Shanghai 200433, China. ${ }^{2}$ China Medical City Institute of Health Sciences, 1 Yaocheng Road, Taizhou, Jiangsu 225300, China. ${ }^{3}$ Eastern Hepatobiliary Surgery Hospital, Second Military Medical University, Shanghai 200438, China. ${ }^{4}$ Department of Health Statistics, Second Military Medical University, Shanghai 200433, China. ${ }^{5}$ National Innovation Alliance for Hepatitis \& Liver Cancer, Shanghai 200438, China. ${ }^{6}$ Cancer Institute, Fudan University Shanghai Cancer Center, Shanghai 200032, China. ${ }^{7}$ Department of Oncology, Fudan University Shanghai Medical College, Shanghai 200032, China.

\section{Received: 2 February 2015 Accepted: 8 March 2016} Published online: 12 March 2016

\section{References}

1. European Association For The Study Of The Liver. EASL clinical practice guidelines: Management of chronic hepatitis B virus infection. J Hepatol. 2012;57(1):167-85.

2. Sjogren M. Immunization and the decline of viral hepatitis as a cause of acute liver failure. Hepatology. 2003;38(3):554-6.

3. McMahon BJ, Holck P, Bulkow L, Snowball M. Serologic and clinical outcomes of 1536 Alaska Natives chronically infected with hepatitis B virus. Ann Intern Med. 2001;135(9):759-68.

4. Toh QC, Tan TL, Teo WQ, et al. Identification of cellular membrane proteins interacting with hepatitis B surface antigen using yeast split-ubiquitin system. Int J Med Sci. 2005:2(3):114-7.

5. Cho DY, Yang GH, Ryu CJ, Hong HJ. Molecular chaperone GRP78/BiP interacts with the large surface protein of hepatitis $B$ virus in vitro and in vivo. J Virol. 2003;77(4):2784-8.

6. Budkowska A, Quan C, Groh F, et al. Hepatitis B virus (HBV) binding factor in human serum: candidate for a soluble form of hepatocyte HBV receptor J Virol. 1993;67(7):4316-22.

7. Yan $H$, Zhong $G, X u G$, et al. Sodium taurocholate cotransporting polypeptide is a functional receptor for human hepatitis $B$ and $D$ virus. Elife. 2012;1:e00049.

8. Zhong $\mathrm{G}$, Yan $\mathrm{H}$, Wang $\mathrm{H}$, et al. Sodium taurocholate cotransporting polypeptide mediates woolly monkey hepatitis B virus infection of Tupaia hepatocytes. J Virol. 2013;87:7176.
9. Drexler JF, Geipel A, Konig A, et al. Bats carry pathogenic hepadnaviruses antigenically related to hepatitis $B$ virus and capable of infecting human hepatocytes. Proc Natl Acad Sci U S A. 2013;110(40):16151-6.

10. Kamatani $Y$, Wattanapokayakit $\mathrm{S}, \mathrm{Ochi} \mathrm{H}$, et al. A genome-wide association study identifies variants in the HLA-DP locus associated with chronic hepatitis B in Asians. Nat Genet. 2009:41(5):591-5.

11. Jiang DK, Sun J, Cao G, et al. Genetic variants in STAT4 and HLA-DQ genes confer risk of hepatitis B virus-related hepatocellular carcinoma. Nat Genet. 2013:45(1):72-5.

12. Li S, Qian J, Yang Y, et al. GWAS identifies novel susceptibility loci on 6p21. 32 and 21q21.3 for hepatocellular carcinoma in chronic hepatitis B virus carriers. PLoS Genet. 2012;8(7):e1002791.

13. Zhang $H$, Zhai $Y, H u$ Z, et al. Genome-wide association study identifies 1 p36.22 as a new susceptibility locus for hepatocellular carcinoma in chronic hepatitis B virus carriers. Nat Genet. 2010:42(9):755-8.

14. Su Z, Li Y, Liao Y, et al. Association of the gene polymorphisms in sodium taurocholate cotransporting polypeptide with the outcomes of hepatitis B infection in Chinese Han population. Infect Genet Evol. 2014;27:77-82.

15. Peng $L$, Zhao Q, Li Q, et al. The p.Ser267Phe variant in SLC10A1 is associated with resistance to chronic hepatitis B. Hepatol. 2015:61(4):1251-60.

16. Dai CY, Yu ML, Chen SC, et al. Clinical evaluation of the COBAS Amplicor HBV monitor test for measuring serum HBV DNA and comparison with the Quantiplex branched DNA signal amplification assay in Taiwan. J Clin Pathol. 2004:57(2):141-5.

17. Hasan KN, Rumi MA, Hasanat MA, et al. Chronic carriers of hepatitis $B$ virus in Bangladesh: a comparative analysis of HBV-DNA, HBeAg/ anti-HBe, and liver function tests. Southeast Asian J Trop Med Public Health. 2002;33(1):110-7.

18. Wang XF, Lu M, Qian J, et al. Rationales, design and recruitment of the Taizhou Longitudinal Study. BMC Public Health. 2009;9:233.

19. Paul E. Cizdziel Domenica Simms, Piotr Chomczynski, TRIzol ${ }^{\mathrm{TM}}$ : a new reagent for optimal single-step isolation of RNA. Focus. 1993;15(4):99-102.

20. Barrett JC, Fry B, Maller J, Daly MJ. Haploview: analysis and visualization of LD and haplotype maps. Bioinformatics. 2005;21(2):263-5.

21. Shiao T, Iwahashi M, Fortune J, et al. Structural and functional characterization of liver cell-specific activity of the human sodium/ taurocholate cotransporter. Genomics. 2000;69(2):203-13.

22. Ng PC, Henikoff S. SIFT: Predicting amino acid changes that affect protein function. Nucleic Acids Res. 2003:31(13):3812-4.

23. Erdman MM, Creekmore LH, Fox PE, et al. Diagnostic and epidemiologic analysis of the 2008-2010 investigation of a multi-year outbreak of contagious equine metritis in the United States. Prev Vet Med. 2011;101(3-4):219-28.

24. Ho RH, Leake BF, Roberts RL, Lee W, Kim RB. Ethnicity-dependent polymorphism in $\mathrm{Na}+$-taurocholate cotransporting polypeptide (SLC10A1) reveals a domain critical for bile acid substrate recognition. J Biol Chem. 2004:279(8):7213-22.

25. Tokuhiro S, Yamada R, Chang X, et al. An intronic SNP in a RUNX1 binding site of SLC22A4, encoding an organic cation transporter, is associated with rheumatoid arthritis. Nat Genet. 2003;35(4):341-8.

26. Lio D, Marino V, Serauto A, et al. Genotype frequencies of the $+874 \mathrm{~T}->\mathrm{A}$ single nucleotide polymorphism in the first intron of the interferon-gamma gene in a sample of Sicilian patients affected by tuberculosis. Eur J Immunogenet. 2002;29(5):371-4

27. Rohrer J, Conley ME. Transcriptional regulatory elements within the first intron of Bruton's tyrosine kinase. Blood. 1998:91(1):214-21.

28. Heinemeyer $T$, Wingender $E$, Reuter I, et al. Databases on transcriptional regulation: TRANSFAC, TRRD and COMPEL. Nucleic Acids Res. 1998;26(1):362-7.

29. Yan H, Peng B, Liu Y, et al. Viral entry of Hepatitis B and D viruses and bile salts transportation share common molecular determinants on sodium taurocholate cotransporting polypeptide. J Virol. 2014;88:3273.

30. Ding X, Mizokami M, Yao G, et al. Hepatitis B virus genotype distribution among chronic hepatitis B virus carriers in Shanghai, China. Intervirology. 2001;44(1):43-7.

31. Zumbika E, Ruan $\mathrm{B}, \mathrm{Xu} \mathrm{CH}$, et al. HBV genotype characterization and distribution in patients with HBV-related liver diseases in Zhejiang Province, P.R. China: possible association of co-infection with disease prevalence and severity. Hepatobiliary Pancreat Dis Int. 2005;4(4):535-43. 